\title{
INFLUÊNCIA PROGNÓSTICA ADVERSA DO DIABETES MELLITUS E DA HIPERGLICEMIA SOBRE A EVOLUÇÃO DO INFARTO CEREBRAL
}

\author{
CHARLES ANDRE * - SERGIO AUGUSTO PEREIRA NOVIS **
}

\begin{abstract}
RESUMO - Em uma série de 109 casos de infarto cerebral comprovado por tomografia computadorizada de crânio, diabetes mellitus esteve presente em 15,6\%. Em 86 casos avaliados precocemente foi detectada hiperglicemia em $67,4 \%$, e em $100 \%$ de 14 pacientes díabéticos. Houve aumento (não significativo) nas taxas de letalidade hospitalar precoce - até o $30^{\circ}$ dia de internação - de pacientes diabéticos; suas chances de obter aita precocemente, porém, pareceram reduzidas. A evolução de pacientes com hiperglicemia também pareceu pior. Estes pacientes exibiram redução significativa das chances de obter alta hospitalar precoce. O risco de vida pareceu máximo em diabéticos hiperglicêmicos $(42,9 \%)$. As aparentes diferenças de letalidade e necessidade de internação prolongada entre pacientes não diabéticos, normo ou hiperglicêmicos, não alcançaram significiação estatística. Os achados do presente estudo parecem indicar uma somação de efeitos deletérios pela hiperglicemia e pelo diabetes no curso do infarto cerebral. Cabe agora a avaliação crítica da sugestão de que a normalização precoce de hiperglicemia poderia levar a redução do grau de lesão cerebral após AVE isquêmico.
\end{abstract}

PALAVRAS-CHAVE: infarto cerebral, diabetes mellitus, hiperglicemia, prognóstico.

Adverse prognostic influence of diabetes mellitus and hyperglycemia on the clinical course of brain infaret.

SUMMARY - We acessed the potential impact of diabetes mellitus and hyperglycemia on early prognosis in a study of 109 cases of CT proved brain infarction. Main end-points of this study were 30 -days case-fatality rates and the probability of early discharge or prolonged hospitalization. Proportions of patients exhibiting diverse evolutions were compared by Fisher's test and, when the number of observations permitted, by chi-square test. Diabetes mellitus was diagnosed by history in $15.6 \%$ (17 patients). In 86 cases analysed within 72 hours of admission, hyperglycemia (glucose level higher than $110 \mathrm{mg} / \mathrm{dL}$ ) was detected in $67.4 \%$ (including all 14 diabetic patients evaluated). A nonsignificant increase in case fatality rates was detected in diabetic patients $(35.3 \%$ vg $21.7 \%$; $\mathrm{p}=0.18)$, but these patients probably were discharged early less frequently $(23.5 \%$ vs $51.1 \% ; \mathrm{p}=0.06)$. Compared to the normoglycemic patients, hyperglycemic patients also fared worse, when considering altogether the early discharge and case fatality rates and the need for prolonged hospitalization $(p=0.06)$. They also exhibited a nonsignificant increase in early case fatality rate $(31 \%$ vs $15.4 \% ; \mathrm{p}=0.21)$ and a markedly reduced chance of obtaining early hospital discharge $(37.9 \%$ vs $65.4 \%$; maximal in diabetic hyperglycemic patients (42.9\%) and seemed intermediate in non-diabetic hyperglycemic patients $(27.3 \%)$. Considering the three end-points evaluated, the apparent differences between non-diabetic hyper and normoglycemic patients did not reach statistical significance $(p=0.19)$. These results suggest that both diabetes mellitus and hyperglycemia «per se» have deleterious effects on the clinical evolution of patients with cerebral infarction. It is now time to consider a prospective study on the potential benefits of early correction of hyperglycemia in these patients.

KEY WORDS: brain infarction, diabetes mellitus, hyperglycemia, prognosis.

Trabalho realizado no Serviço de Neurologia do Hospital Clementino Fraga Filho da Universidade Federal do Rio de Janeiro (HUCFF-UFRJ): * Médico Neurologista, Serviço de Neurologia, HUCCF-UFRJ; ** Professor Titular de Neurologia, Chefe do Servico de Neurologia, HUCFF-UFRJ.

Dr. Sérgio A. Pereira Novis - Rua Visconde de Piraja 330, sala 513 - 22410 Rio de Janeiro RJ - Brasil. 
Diversos estudos sugerem que a presença de hiperglicemia pode contribuir para um aumento da extensão da lesão e taxa de letalidade por infarto cerebral (IC). Esta impressão, porém, não alcança ainda aceitação uniforme e poucos estudos utilizaram-se rotineiramente de métodos neurorradiológicos confiáveis para a confirmação diagnóstica. O potencial efeito deletério do diabetes mellitus (DM) sobre a evolução inicial destes pacientes, igualmente, permanece em debate. Neste estudo, procurou-se avaliar o impacto adverso desses dois fatores sobre a evolução hospitalar em pacientes com IC confirmado por tomografia computadorizada (TC).

\section{MATERIAIS E MÉTODOS}

Entre 1986 e 1987, 109 casos de IC, ocorrendo em 108 adultos, foram estudados no HUCFF-UFRJ na fase mais aguda de sua doença - admissão hospitalar até o sétimo dia de evolução. Os pormenores de composição da casuística foram previamente esmiuçados 2. Todos os pacientes foram submetidos a um ou mais exames por TC durante a internação. viagnósticos alternativos foram sistematicamente excluídos, empregando-se critéríos clínicos e tomográficos rígidos de seleção 2 .

Quanto ao diagnóstico prévio de DM, apenas dados clínicos efetivamente referidos à anamnese foram considerados. Somente amostras de sangue obtidas precocemente (até 72 horas após a admissão) foram consideradas para análise da glicemia e de outros parâmetros bioquímicos e hematológicos 2. As amostras foram analisadas pelo método da glicose-oxidase (GOD-PAP), sendo considerados normais valores entre 70 e $110 \mathrm{mg} / \mathrm{dL}$.

Foram analisadas especialmente as taxas de letalidade hospitalar precoce - até o $30^{\circ}$ dia de internaçāo - e as chances de obter alta hospitalar precocemente. As proporções de pacientes apresentando diversas evoluções foram comparadas pelos métodos de Fisher e, quando o número de observações permitiu, o do qui-quadrado $\left(\chi^{2}\right)$. 'Os testes foram selecionados por programa estatístico computadorizado largamenté utilizado (EPISTAT r).

\section{RESULTADOS}

Diagnóetico prévio de DM foi referido em 17 casos $(15,6 \%)$. A glicemia fol avaliada precocemente em 86 casos (78,9\%). Hiperglicemia foi detectada em 58 casos $(67,4 \%)$. Enquanto todos os 14 pacientes diabéticos avaliados exibiam hiperglicemia, este percentual caiu a $61,1 \%$ entre não diabéticos. Dois pacientes apresentando hipoglicemia foram exclúdos da análise.

Seis pacientes com DM faleceram nos primeiros 30 dias de internação. A comparação de sua taxa de letalidade $(35,3 \%)$ com a do grupo de não diabéticos $(21,7 \%)$ não revelou diferença estatisticamente significativa (Fisher, $p=0,183$ ). Por outro lado, as chances de obter alta precocemente pareceram menores em diabéticos: $23,5 \%$ vs $51,1 \%\left(\chi^{2}, \mathrm{p}=0,067\right)$.

A comparação global entre pacientes com ou sem hiperglicemia sugere fortemente um agravamento clínico em pacientes hiperglicêmicos (Tabela 1). O aparente aumento da taxa de letalidade neste grupo não é confirmado estatisticamente $\left(\chi^{2}, p=0,215\right)$, mas foi claramente demonstrada sua reduzida chance de obter alta hospitalar precocemente $\left(\chi^{2}, \mathrm{p}=0,036\right)$. A taxa de letalidade em pacientes normoglicêmicos pode ser considerada baixa.

Tabela 1. Indicadores prognósticos em pacientes com infarto cerebral segundo o nivel inicial de glicemia: $\%(n)$.

\begin{tabular}{|c|c|c|c|c|c|}
\hline $\begin{array}{l}\text { Grupo } \\
\text { estudado }\end{array}$ & Glicemia & $\begin{array}{l}\text { Mortialidade } \\
\text { precoce }\end{array}$ & $\begin{array}{c}\text { Alta } \\
\text { precoce }\end{array}$ & $\begin{array}{c}\text { Hospitalizaçăo } \\
\text { prolongada }\end{array}$ & $\begin{array}{r}\text { Significacão } \\
\text { estatística }\end{array}$ \\
\hline \multirow[t]{4}{*}{$\begin{array}{r}\text { Total } \\
(86)\end{array}$} & $\begin{array}{l}\text { Normal } \\
(26)\end{array}$ & $15,4(4)$ & $19,2(5)$ & 65,4 (17) & $x^{2} p=0,063$ \\
\hline & $\begin{array}{l}\text { Hiperglicemia } \\
\text { (58) }\end{array}$ & $31 \quad$ (18) & $31 \quad$ (18) & $37,9(22)$ & \\
\hline & $\begin{array}{l}\text { Não-diabéticos com } \\
\text { hiperglicemia }(44)^{*}\end{array}$ & 27,3 (12) & 29,6 (13) & 43,2 (19) & $\chi^{2} p=0,196$ \\
\hline & $\begin{array}{l}\text { Diabéticos com } \\
\text { hiperglicemia (14) }\end{array}$ & $42,9(6)$ & $35,7(5)$ & $21,4(3)$ & \\
\hline
\end{tabular}

* urupo comparado diretamente aquele formado por 26 pacientes não-diabéticos normoglicêmicos. 
O grupo com maior risco de vida e menores chances de obter alta precocemente foj o de diabéticos hiperglicêmicos: 42,9\%. A comparação direta deste grupo com pacientes normoglicêmicos, porém, é temerária, já que normoglicemia só foi encontrada entre pacientes sem DM. Todos os óbitos entre pacientes diabéticos deram-se naqueles em que foi demonstrada hiperglicemia.

Considerando apenas pacientes não diabéticos, a comparação global de pacientes com e sem hiperglicemia não demonstra diferenças prognósticas significativas (Tabela 1). Suas taxas de letalidade são comparáveis $\left(\chi^{2}, \quad \mathrm{p}=0,395\right)$. As chances de pacientes híperglicêmicos receberam alta precoce mostraram-se aparentemente reduzidas, sem que a diferença detectada alcançasse significação estatística $\left(\chi^{2,}, \quad p=0,121\right)$.

\section{COMENTARIOS}

A prevalência de DM detectada no presente estudo é semelhante à de estudos prévios 10,13,18,20. O achado de hiperglicemia em todos os pacientes diabéticos, logo após o icto, não surpreende. Mais interessante é a grande incidência da alteração entre indivíduos sem diagnóstico prévio. É possível que fração destes constituísse um grupo de diabéticos até então não reconhecidos. De todo modo até as mais discretas elevações glicêmicas foram sempre computadas. Em alguns estudos prévios 4,6,15,17,19, a presença de DM associou-se a aumento da mortalidade por AVE. Este achado, porém, não é uniforme, havendo várias séries nas quais não se detectou agravamento prognóstico por AVE ou, especificamente, por IC 1,7-9,12,14,18. No presente estudo, apenas uma redução limiarmente significativa das chances de obter alta precoce foi detectada entre diabéticos, o que pode sugerir maior incidência de complicações clínicas tratáveis.

A história de DM correlacionou-se intimamente ao achado de hiperglicemia à internação. A taxa de letalidade entre diabéticos com hiperglicemia foi de $42,9 \%$, aparentemente superior à de não diabéticos hiperglicêmicos (27,3\%). Estes dados, acrescidos da redução da probabilidade de obter alta, aproximam os resultados desta série aos de Caronna e Levy 7, que sugeriram a existência de efeito adverso da hiperglicemia apenas em pacientes diabéticos. A ausência de diabéticos normoglicêmicos à internação impede crítica direta à sugestão de Candelise e col.6 de que 0 DM somente associa-se a aumento do risco de vida na presença de hiperglicemia. As causas da associação entre o DM e pior evolução clínica no curso do IC são revistas pelos autores em outra publicação 3 .

Já em 1956, Daalsgaard-Nielsen 9 sugeria que o achado de glicemias elevadas, acima de $110 \mathrm{mg} / \mathrm{dL}$, durante a internação implicava em maior taxa de letalidade por AVE em geral. Estudos subseqüentes pareceram confirmar esta impressão, ao detectar efeito deletério no curso do IC $7,15,21,22$, apesar do surgimento esporádico de resultados contraditórios 12. Parece provável, ainda, que este efeito adverso não se restrinja ao âmbito dos AVE isquêmicos, podendo manifestar-se também após hemorragias cérebro-meníngeas 6,22 .

Os resultados da presente série vêm somar-se à literatura já extensa sobre a importância prognóstica da hiperglicemia no curso do IC. A presença de hiperglicemia assuciou-se fortemente às mortes por quaisquer mecanismos, estando presente em mais de $70 \%$ dos casos de óbito por deterioração neurológica progressiva, sepse ou complicações cardiovasculares. Nossos resultados sugerem, ainda, que o efeito deletério da hiperglicemia não se restringe apenas aos pacientes não-diabéticos, como sugerido por Candelise e col.6. Pelo contrário, neste grupo, as diferenças detectadas na evolução global e a redução das chances de obter alta precocemente, apesar de ainda aparentes, não mais alcançam significação estatística.

CONCLUSAO - Considerando diversos parâmetros, os achados do presente estudo são semelhantes aos de Caronna e Levy 7 e parecem indicar uma somação de efeitos deletérios da própria hiperglicemia e das alterações prévias induzidas pelo DM. Os mecanismos potenciais de agravamento da lesão isquêmica e da evolução clínica nestas circunstâncias são revistos em publicação complementar 3 .

Pulsinelli, cm mais de um artigo 15,16 , sugere que a normalização precoce da hiperglicemia poderia diminuir o grau de lesão cerebral no AVE isquêmico. Esta suposição, entretanto, ainda não foi testada rigorosamente e a extrapolação de dados experimentais para situaçōes clínicas reais deve ser sempre cautelosa. De todo modo, 
há evidências preliminares de que a administração de insulina a animais hiperglicêmicos, em modelos de isquemia cerebral, pode levar a menor lesão neurológica e melhor prognóstico, segundo Helgason 11, bem como Siemkowicz e col. (citados por Berger e Hakim 5 ).

\section{REFERENCIAS}

1. Allen CMC. Predicting the outcome of acute stroke: a prognostic score. $J$ Neurol Neurosurg Psychiatry 1984, 47:475-480.

2. André C. A morte na fase aguda do infarto cerebral: Cronologia e causas de óbito determinantes prognósticos adversos. Tese, INDC-UFRJ. Rio de Janeiro, 1991.

3. André C, Novis SAP. Influência prognóstica adversa do diabetes mellitus e da hiperglicemia sobre a evolução do infarto cerebral: uma revisão de suas causas, com menção aos mecanismos de gênese da hiperglicemia na fase aguda do AVE. Arq îeuro-Psiquiat (São Paulo) 1992, 50:151-155.

4. Asplund K, Häag E, Helmers C, Lithner F, Strand T, Wester PO. The natural history of stroke in diabetic patients. Acta Med Scand 1980, 207:417-424.

5. Berger L, Hakim AM. The association of hyperglycemia with cerebral edema in stroke. Stroke 1986, 17:865-871.

6. Candelise L, Landi G, Orazio EN, Bocciardi E. Prognostic significance of hyperglycemia in acute stroke. Arch Neurol 1985, 42:661-663.

7. Caronna JJ, Levy DE. Clinical predictors of outcome in ischemic stroke. Neurologic Clinics 1983, 1:103-117.

8. Chambers BR, Norris JH, Schurvell BL, Hachinski VC. Prognosis of acute stroke. Neurology 1987, 37:221--225.

9. Daalsgard-Nielsen $T$. Some clinical experience in the treatment of cerebral apoplexy (1000 cases). Acta Psychiat Scand 1956, Suppl, 108:101.

10. Harmsen P, Tibbling G. A stroke register in Göteborg, Sweden. Acta Med Sctand 1972, 191:463-470.

11. Helgason CM. Blood glucose and stroke. Strok 1988, 19:1049-1053.

12. Miah K, Arbin MV, Britton M, Faire U, Helmers C, Maasing $R$. Prognosis in acute stroke with special reference to some cardiac factors. J Chron Dis 1983, 36:279-288.

13. Mohr JP, Caplan LR, Melski JW et al. The Harvard cooperative stroke registry: a prospective registry. Neurology 1978, 28:754-762.

14. Patrick BK, Ramirez-Lasseras M, Snyder BD. Temporal profile of vertebrobasilar territory infarction: prognostic implications. Stroke 1980, 11:643-648.

15. Pulsinelli WA, Levy DE, Sigsbee $B$ et al. Increased damage after ischemic stroke in patients with hyperglycemia with or without established diabetes mellitus. Am J Med 1983, 74:540-544.

16. Puisinelli WA, Waldman S, Rawlinson D, Plum F. Moderate hyperglycemia augments ischemic brain damage: a neuropathologic study in the rat. Neurology 1982, 32:1239-1246.

17. Pulsinelli WA, Waldman S, Sigsbee B, Rawlinson D, Scherer P, Plum F. Experimental hyperglycemia and diabetes mellitus worsen stroke outcome. Trans Am Neurol Assoc 1980, $105: 21-24$.

18. Robinson RW, Cohen WD, Higano $N$ et al. Analysis of survival after cerebral thrombosis: ten-year experience. JAMA 1959, 169:1149-1152.

19. Robinson RW, Demirel M, LeBeau RJ. Natural history of cerebral thrombosis: nine to nineteen year follow-up. J Chron Dis 1968, 21:221-230.

20. Térent A, Anderson $B$. The prognosis for patients with cerebrovascular stroke and transient ischemic attacks. Upsala J Med Sci 1981, 86:63-74.

21. Woo E, Chan YW, Yu YL, Huang CY. Admission glucose level in relation to mortality and morbidity outcome in 252 stroke patients. Stroke 1988, 19:185-191.

22. Woo E, Ma JTC, Robinson JD, $Y u$ YL. Hyperglycemia is a stress response in acute stroke. Stroke 1988, 19:1359-1364. 